\title{
Electromagnetically induced transparency in terahertz plasmonic metamaterials via dual excitation pathways of the dark mode
}

\author{
Xiaojun Liu (刘小君), ${ }^{1}$ Jianqiang Gu (谷建强), ${ }^{1, \text { a) }}$ Ranjan Singh, ${ }^{2, \mathrm{~b})}$ Yingfang Ma (马颖芳), ${ }^{1}$ \\ Jun Zhu (朱俊), ${ }^{1}$ Zhen Tian (田震), ${ }^{1}$ Mingxia He (何明霞), ${ }^{1}$ Jiaguang Han (韩家广), ${ }^{1, c)}$ \\ and Weili Zhang (张伟力) $)^{1,2}$ \\ ${ }^{1}$ Center for Terahertz waves and College of Precision Instrument and Optoelectronics Engineering, Tianjin \\ University, and the Key Laboratory of Optoelectronics Information Technology (Ministry of Education), \\ Tianjin 300072, People's Republic of China \\ ${ }^{2}$ School of Electrical and Computer Engineering, Oklahoma State University, Stillwater, Oklahoma 74078, \\ USA
}

(Received 20 December 2011; accepted 5 March 2012; published online 26 March 2012)

\begin{abstract}
We observe the excitation and tuning of electromagnetically induced transparency (EIT) by the interference between different excitation pathways of the dark mode in a planar terahertz metamaterial. The EIT unit cell consists of a cut wire as the bright resonator and a pair of split ring resonators (SRRs) as the dark element. The dark mode resonance is excited by both the electric and magnetic fields when the SRR pair translates along the wire without altering the lateral distance between the resonators. The electric and magnetic pathways of exciting the dark mode allows for a giant amplitude modulation of the EIT resonance. (C) 2012 American Institute of Physics. [http://dx.doi.org/10.1063/1.3696306]
\end{abstract}

Electromagnetically induced transparency (EIT) is a quantum interference effect that occurs in a laser-driven atomic system leading to an extremely narrowband transparency window over a wide absorption spectrum. ${ }^{1}$ The effect dramatically modifies the dispersive properties of the original opaque medium and enables light pulses to be slowed down by $\sim 7$ orders of magnitude. ${ }^{2}$ However, limited material options and stringent requirements to preserve coherence of the excitation pathways in atomic systems have significantly constrained further investigations and realistic applications of the EIT effect. Recent studies have shown that the EIT effect can be mimicked by non-quantum approaches, such as onchip plasmonic structures and photonic resonators. ${ }^{3}$ In particular, metamaterial induced transparency in subwavelength structures, such as split ring resonators (SRRs), dipolequadruple coupled systems, Fano resonators, and multi-layer fish scales are the most recent and promising additions to the existing classical EIT schemes. ${ }^{4-18}$ Being treated as an effective media, the EIT metamaterial resonators are considered as a promising candidate in realizing bulk slow light devices. Furthermore, the sharp EIT-like resonance in metamaterials can be exploited as promising sensing devices. ${ }^{12}$ Thus, there is enormous interest recently in the classical EIT phenomena due to its multifunctional applications.

In general, the EIT-like spectral response is a result of destructive interference between the bright and dark mode resonances. $^{4-11}$ The bright mode usually possesses a low quality factor $(Q)$ due to its superradiant nature and the dark mode has a high $Q$ since it is subradiant. Previous studies have shown that manipulating the EIT effect could be realized by breaking the symmetry of the unit cell structure or

\footnotetext{
a)Electronic mail: gjq@tju.edu.cn.

${ }^{\text {b) }}$ Present address: Center for Integrated Nanotechnologies, Materials Physics and Applications Division, Los Alamos National Laboratory, Los Alamos, New Mexico 87545, USA.

${ }^{c)}$ Electronic mail: jghan@tju.edu.cn.
}

by varying the relative distance between the resonators, thus modifying the coupling between the bright and dark modes. ${ }^{4-6}$ Very limited attempts, however, have been made to mimic the EIT phenomena without either breaking the structural symmetry or by varying the lateral distance between the resonators. In this letter, we show that the EIT effect can be resonantly excited in a symmetric structure without altering the separation between the bright and dark mode resonators. A significant amplitude modulation of the EIT resonance is demonstrated by translating the dark resonator pair along the fixed bright resonator. This allows for the manipulation of the interference between different excitation pathways of the dark mode as well as controls the coupling between the dark and the bright mode resonators.

The metamaterial unit cell consists of a pair of SRRs symmetrically placed on the left and right side of a cut wire, as illustrated in Fig. 1(a). The 200-nm-thick aluminum metamaterial samples were fabricated on a $640-\mu$ m-thick n-type silicon substrate by conventional optical lithography. The high resistivity silicon substrate is non-dispersive and has low absorption at terahertz frequencies. ${ }^{19}$ The carrier concentration in the $\mathrm{n}$ type substrate is $\sim 4.4 \times 10^{14} \mathrm{~cm}^{-3}$ and the resistivity value is $\sim 12 \mathrm{Ohm} / \mathrm{cm}$. The amplitude transmission spectra of the samples were measured by an 8-F confocal terahertz time-domain spectroscopy (THz-TDS) system. ${ }^{19,20}$

The incident electric field $\boldsymbol{E}$ is oriented along the $y$ axis. Figure 1(b) shows a sole-wire array (left inset) that exhibits a typical localized surface plasmon (LSP) resonance at $0.67 \mathrm{THz}$ (solid curve). However, in this case, the LC resonance in the sole-SRR pair (right inset) cannot be excited directly due to the structural symmetry with respect to the exciting field. With $\boldsymbol{E}$ along the $x$ axis, on the other hand, a sharp LC resonance is easily excited in the sole SRR pair (dotted curve) at exactly the same frequency as that of the LSP resonance in the wire array. As a result, when these two types of resonators are coupled together by arranging them in close proximity to each other within a unit cell, a typical EIT like 


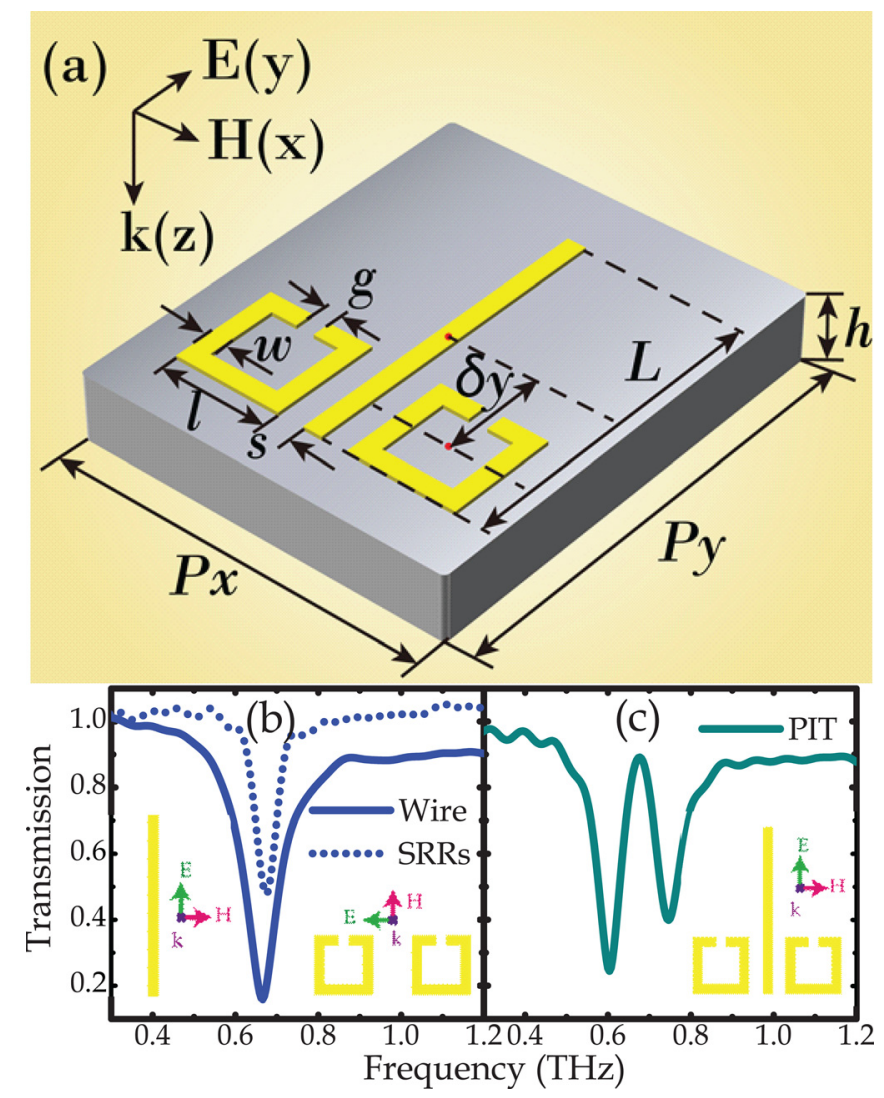

FIG. 1. (Color online) (a) Schematic diagram of the EIT metamaterial structure with geometrical parameters: $P x=80 \mu \mathrm{m}, P y=120 \mu \mathrm{m}, L=85 \mu \mathrm{m}$, $l=29 \mu \mathrm{m}, s=7 \mu \mathrm{m}, g=5 \mu \mathrm{m}, w=5 \mu \mathrm{m}$, and $\delta y=-28 \mu \mathrm{m}$. Measured amplitude transmissions for (b) sole-wire and sole-SRR pair pattern, and (c) EIT metamaterial comprised of wire and SRR pair in the unit cell.

spectral response is observed with the $y$ polarized $\boldsymbol{E}$ field excitation, as shown in Fig. 1(c). In this design, the wire array can be excited directly by the incident electric field, acting as the bright resonator, while the fundamental LC resonance in the SRRs cannot be excited by the incident $\boldsymbol{E}$ field, thus acting as the dark mode. It is the destructive interference between the bright and dark resonators that gives rise to a strong EIT effect, leading to a sharp transparency window at $0.67 \mathrm{THz}$.

The amplitude modulation of the transparency peak is observed by displacing the SRR pair vertically along the wire, where the symmetry of the whole structure along the $y$ axis is unperturbed and the wire-SRR lateral distance along the $x$ axis remains constant as well. In our experiment, it is surprising to see the giant amplitude modulation of the EIT transmission spectra as the SRR pair is gradually displaced from $\delta y=-28$ to $28 \mu \mathrm{m}$, as depicted in Figs. 2(a)-2(d). When the SRRs move upward from $\delta y=-28$ to $12 \mu \mathrm{m}$, the transparency window gradually shrinks without notable frequency shift. Finally, with a further displacement of $\delta y=28 \mu \mathrm{m}$, the EIT feature completely disappears, leaving behind a broad single resonance dip approaching a minimum transmission of 0.13 . The measured tunable spectral response is further supported by full wave numerical simulation using CST Microwave Studio, as shown in Figs. 2(e)-2(h). The simulations are in a good agreement with the measured experimental data. This position-dependent modulation of the amplitude influences the group velocity and thus the slow light performance of the EIT metamaterial. ${ }^{4,7}$ Using the

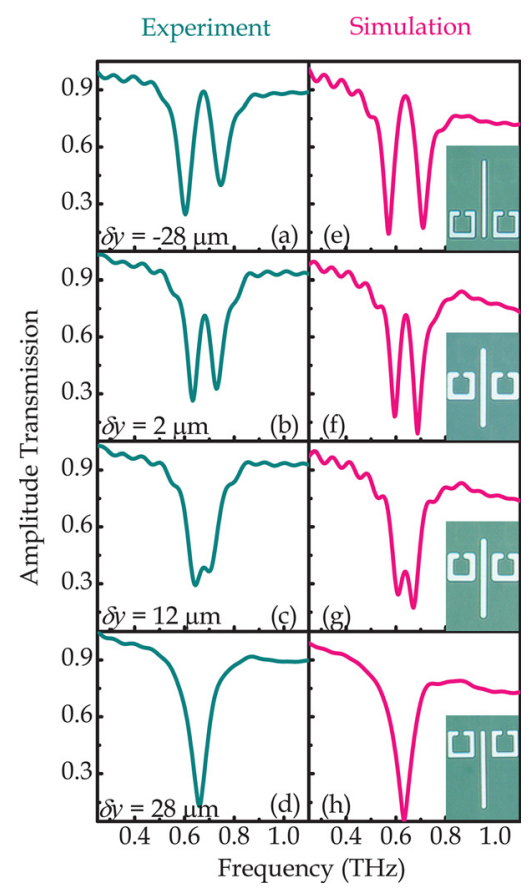

FIG. 2. (Color online) (a)-(d) Experimental transmission spectra and (e)-(h) corresponding simulated spectra for the SRR pair displacements of $\delta y=-28,-2,12$, and $28 \mu \mathrm{m}$, respectively. Inset: microscopic images of the unit cells with different $\delta y$.

measured transmission results, we retrieved the frequency dependent group refractive indices $\left(n_{\mathrm{g}}\right)$ for different SRR positions. The group index for $\delta y=-28 \mu \mathrm{m}$ has the value of 24 at the peak of the EIT transparency window. From the experimental and simulation results, it is clear that the LC resonance in the SRR pair is excited by the near field coupling of the bright mode (wire) to the dark mode (SRR pair). In order to better understand the underlying near field coupling mechanism in the wire-SRR pair system, we further studied two additional cases, i.e., $\delta y=-52$ and $-12 \mu \mathrm{m}$. When $\delta y=-52 \mu \mathrm{m}$, the gap of the SRRs is located parallel to the bottom edge of the bar in the $x$ axis direction, while with $\delta y=-12 \mu \mathrm{m}$, the gap arms of the SRRs are on the same horizontal line that coincides exactly with the center of the wire length. Both cases exhibit the EIT behavior, as shown in Figs. 3(a) and 3(b), respectively. Figure 3(c) shows the simulated distribution of $E_{x}^{\text {Wire }}$ ( $x$ component of the electric field from the wire) and Fig. 3(d) shows $H_{z}^{\text {Wire }}$ ( $z$ component of the magnetic field from the wire), indicating that the dark mode excitation mechanism via the bright mode in samples with $\delta y=-52 \mu \mathrm{m}$ and that with $\delta y=-12 \mu \mathrm{m}$ are different. At $\delta y=-52 \mu \mathrm{m}, H_{z}^{\text {Wire }}$ penetrating the SRRs is too weak to excite the dark LC resonance. The gap of the SRR is near the lower edge of the wire, where $E_{x}^{\text {Wire }}$ has the strongest electric field concentration as it can be seen from the simulated fields in Fig. 3(c). Thus, we believe that the LC dark mode is mainly excited by the strong electric field coupling from the wire edge to the SRR pair. On the other hand, in the case of $\delta y=-12 \mu \mathrm{m}$, the dark mode excitation occurs via the magnetic field of the wire, as seen in the simulated $H_{z}^{\text {Wire }}$ in Fig. 3(d). As the SRR pair is translated along the wire starting from the bottom, the coupling mechanism switches from being capacitive in nature via the electric field to being 


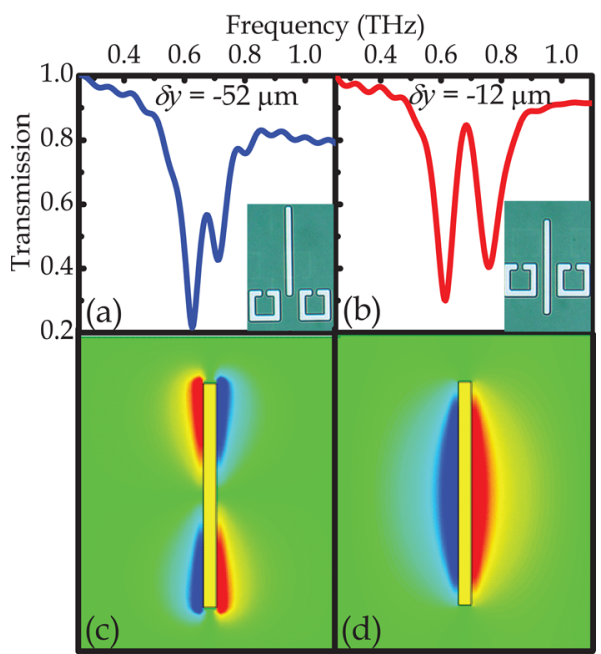

FIG. 3. (Color online) Measured amplitude transmission spectra of the EIT structures for (a) $\delta y=-52 \mu \mathrm{m}$, (b) $\delta y=-12 \mu \mathrm{m}$ with the microscopic images of the corresponding unit cell inserted as the insets, respectively. Simulated distributions of (c) $x$ component of electric field $E_{x}^{\text {Wire }}$ and (d) $z$ component of magnetic field $H_{z}^{\text {Wire }}$ of the sole-wire pattern at $0.67 \mathrm{THz}$.

inductive via the magnetic field of the wire, leading to the observed amplitude modulation of the transparency peak, as shown in Fig. 2. It is worth noting that the amplitude and phase of these two excitation mechanisms alternate as the SRR pair moves upwards along the wire.

To elucidate this interaction of the electrical and magnetic excitation pathways in further detail, surface current distributions of the electrically and magnetically excited LC resonances are shown in Fig. 4(a) for samples with $\delta y=-28$ and $28 \mu \mathrm{m}$ due to their opposite behavior. The surface currents in the sample with $\delta y=-28 \mu \mathrm{m}$ excited by $H_{z}^{\text {Wire }}$ (solid

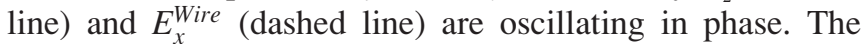
constructive interference between these two surface currents leads to a strong excitation of the LC resonance in the SRRs, enhancing the coupling between the dark and bright modes. However, when the SRRs move to $\delta y=28 \mu \mathrm{m}$, the magnetically stimulated surface current remains constant but the electrically excited current changes its direction according to the distributions of $E_{x}^{\text {Wire }}$ and $H_{z}^{\text {Wire }}$, as shown in Figs. 3(c) and 3(d), respectively. Thus, these two surface currents interact destructively to cancel each other and suppress the excitation of the LC resonance that eventually leads to the disappearance of the EIT peak.

The simulated electric field energy distribution at 0.67 THz shown in Figs. 4(b)-4(d) also fall in line with our interpretation. The EIT resonance effect is most pronounced at $\delta y=-28 \mu \mathrm{m}$ since maximum electric field energy is coupled from the wire to the SRRs once the wire is directly excited by the incident terahertz field oscillating along the wire, as can be seen in Fig. 4(b). At position $\delta y=28 \mu \mathrm{m}$, the SRRs are in a dormant state as all the electric energy is confined around the ends of the bar and such field distribution fails to induce circular currents in the SRRs (Fig. 4(c)). By looking at Figs. 4(c) and 4(d), we could conclude that at $\delta y=28 \mu \mathrm{m}$ the metamaterial structure indeed supports only the LSP resonance since there is no excitation in the SRRs. The EIT peak modulation, as shown in Fig. 2, depends on how well the bright mode in the wire couples to the dark mode of the

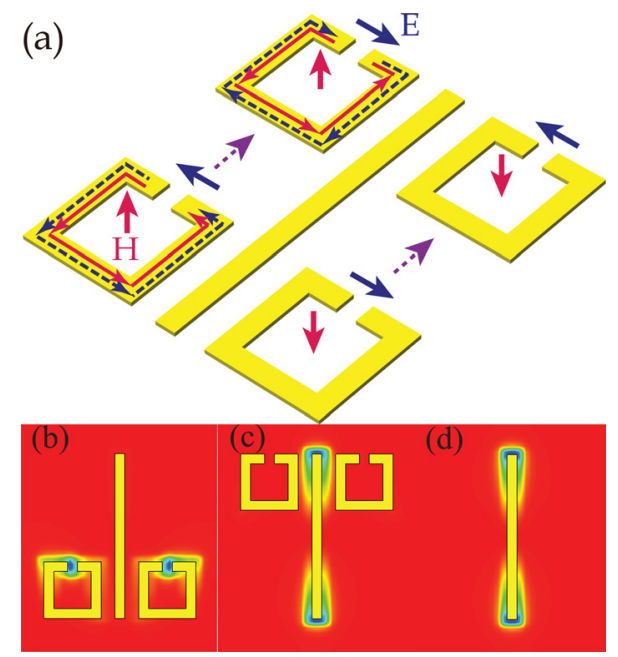

FIG. 4. (Color online) (a) Surface current distributions of the two excitation pathways on SRRs at $\delta y=-28$ and $28 \mu \mathrm{m}$. Electric field energy distributions of the EIT structure for (b) $\delta y=-28 \mu \mathrm{m}$, (c) $\delta y=28 \mu \mathrm{m}$, and (d) that of the sole-wire pattern at $0.67 \mathrm{THz}$.

SRR pair and this coupling in turn depends on the phase difference between the magnetic, $H_{z}^{\text {Wire }}$ and the electric, $E_{x}^{\text {Wire }}$ excitations in the wire. According to the Lorentz oscillation model, there is a $\pi / 2$ phase difference between $H_{z}^{\text {Wire }}$ and $E_{x}^{\text {Wire }}$. The Maxwell equations predict that the magnetically induced surface current is in phase with the magnetic field, while the electrically induced surface current lags by $\pi / 2$ with respect to the electric field. Thus the surface current induced by $H_{z}^{\text {Wire }}$ and $E_{x}^{\text {Wire }}$ will have a phase difference of $\pi$ or 0 , which leads to the destructive or the constructive interference between the surface currents.

In summary, we investigated a unique EIT effect in terahertz metamaterials arising from the interference between different excitation pathways of the dark mode. The structure consists of an SRR pair separated by a wire along the non gap arms of the SRRs. The external incident field oriented along the wire excites the LSP resonance in the wire and the SRR pair is either excited by the electric field of the wire or its magnetic field leading to an EIT type of resonance effect in the transmission spectra. Translating the SRRs along the wire results in a tunable behavior of the EIT resonance due to the constructive or the destructive interference between the electric and the magnetic excitation pathways of the LC resonance in the SRRs. This metamaterial design that enables excitation and tuning of the classical EIT effect would eventually lead to the development of electromagnetic slow light devices.

This work was partially supported by the National Science Foundation of China (Grant Nos. 61107053, 61138001, 61028011, 61007034, 60977064, and 61107085) and the U.S. National Science Foundation.

${ }^{1}$ S. E. Harris, Phys. Today 50, 36 (1997).

${ }^{2}$ L. V. Hau, S. E. Harris, Z. Dutton, and C. H. Behroozi, Nature 397, 594 (1999).

${ }^{3}$ M. F. Yanik, W. Suh, Z. Wang, and S. Fan, Phys. Rev. Lett. 93, 233903 (2004).

${ }^{4}$ S. Zhang, D. A. Genov, Y. Wang, M. Liu, and X. Zhang, Phys. Rev. Lett. 101, 047401 (2008).

${ }^{5}$ N. Papasimakis, V. A. Fedotov, N. I. Zheludev, and S. L. Prosvirnin, Phys. Rev. Lett. 101, 253903 (2008). 
${ }^{6}$ R. Singh, C. Rockstuhl, F. Lederer, and W. Zhang, Phys. Rev. B 79, 085111 (2009).

${ }^{7}$ P. Tassin, L. Zhang, T. Koschny, E. N. Economou, and C. M. Soukoulis, Phys. Rev. Lett. 102, 053901 (2009).

${ }^{8}$ N. Liu, L. Langguth, T. Weiss, J. Kastel, M. Fleischhauer, T. Pfau, and H. Giessen, Nature Mater. 8, 758 (2009).

${ }^{9}$ V. A. Fedotov, M. Rose, S. L. Prosvirnin, N. Papasimakis, and N. I. Zheludev, Phys. Rev. Lett. 99, 147401 (2007).

${ }^{10}$ R. Singh, I. A. I. Al-Naib, M. Koch, and W. Zhang, Opt. Express 19, 6312 (2011).

${ }^{11}$ S.-Y. Chiam, R. Singh, C. Rockstuhl, F. Lederer, W. Zhang, and A. A. Bettiol, Phys. Rev. B 80, 153103 (2009).

${ }^{12}$ Z. G. Dong, H. Liu, J. X. Cao, T. Li, S. M. Wang, S. N. Zhu, and X. Zhang, Appl. Phys. Lett. 97, 114101 (2010).
${ }^{13}$ R. D. Kekatpure, E. S. Barnard, W. Cai, and M. L. Brongersma, Phys. Rev. Lett. 104, 243902 (2010).

${ }^{14}$ Y. Lu, J. Y. Rhee, W. H. Jang, and Y. P. Lee, Opt. Express 18, 20912 (2010).

${ }^{15}$ H. Xu, Y. Lu, Y. Lee, and B. S. Ham, Opt. Express 18, 17736 (2010).

${ }^{16}$ Z. Li, Y. Ma, R. Huang, R. Singh, J. Gu, Z. Tian, J. Han, and W. Zhang, Opt. Express 19, 8912 (2011).

${ }^{17}$ Y. Ma, Z. Li, Y. Yang, R. Huang, R. Singh, S. Zhang, J. Gu, Z. Tian, J. Han, and W. Zhang, Opt. Mater. Express 1, 391 (2011).

${ }^{18}$ C. Wu, A. B. Khanikaev, and G. Shvets, Phys. Rev. Lett. 106, 107403 (2011).

${ }^{19}$ D. Grischkowsky, S. Keiding, M. v. Exter, and C. Fattinger, J. Opt. Soc. Am. B 7, 2006 (1990).

${ }^{20}$ W. Zhang, Eur. Phys. J. Appl. Phys. 43, 1 (2008). 